\title{
Cutaneous manifestations associated with antiphospholipid antibodies in patients with suspected primary antiphospholipid syndrome: a case-control study
}

Luigi Naldi, Francesco Locati, Lorenzo Marchesi, Sergio Cortelazzo, Guido Finazzi, Monica Galli, Aldo Brevi, Tullio Cainelli, Tiziano Barbui

\begin{abstract}
Objective To study the association of a variety of dermatological manifestations related to vascular abnormalities with antiphospholipid antibodies in patients with suspected primary antiphospholipid syndrome.

Method Case-control study. Consecutive patients referred to the coagulation and haemostasis service of a general hospital for the first determination of antiphospholipid antibodies (lupus anticoagulant and anticardiolipin antibodies) and newly diagnosed disorders (for example, thrombocytopenia, thrombotic disorders, and unexplained repeated abortions) were selected. Patients were examined by two dermatologists according to predefined criteria, and information about general characteristics and relevant dermatological and medical histories were collected using an ad hoc questionnaire. The study was limited to patients without evidence of systemic lupus erythematosus. A total of 35 patients was examined; 13 subjects were positive for lupus anticoagulant or anticardiolipin antibodies, or both (cases), and 22 were negative (controls).
\end{abstract}

Results Moderate to severe livedo reticularis and acrocyanosis were significantly associated with antiphospholipid antibodies, with relative risks of $13.1(95 \%$ confidence interval 1.1 to 149.0$)$ and $8.6(95 \%$ confidence interval $1 \cdot 1$ to $65 \cdot 1)$. Capillaritis was also associated with the antibodies. Histories of Raynaud's phenomenon and superficial thrombophlebitis were more common in cases than controls.

Conclusions This study provides quantitative evidence of the association of antiphospholipid antibodies with several cutaneous diseases in which vascular abnormalities seem to play a major part. The study suggests that these manifestations might appear early in the development of the antiphospholipid syndrome.

(Ann Rheum Dis 1993; 52: 219-222)

Lupus anticoagulant and anticardiolipin antibodies, collectively called antiphospholipid antibodies, are closely related autoantibodies that can be found in patients with systemic lupus erythematosus (SLE) and also in patients without evidence of SLE, some with other autoimmune, infectious, or neoplastic diseases, or receiving some drugs (for example, hydralazine). ${ }^{1}$ Antiphospholipid antibodies have been strongly associated with certain clinical manifestations, including arterial and venous thrombotic disorders, repeated abortion, and thrombocytopenia, which together comprise the so called 'antiphospholipid syndrome'. ${ }^{3}$ Most of the evidence was initially derived from case series of highly selected patients ${ }^{4}$ and, notably, in addition to confirmatory data, ${ }^{56}$ partly divergent results ${ }^{7}$ have been obtained when more formal methodologies such as epidemiological studies were applied. Prospective cohort studies and cross sectional studies of unselected patients presenting with their first clinical manifestations have been strongly advocated ${ }^{4}$ to obtain better estimates of the associations in the average patient. A variety of cutaneous disorders, ${ }^{89}$ principally related to vascular abnormalities, such as livedo reticularis and several types of necrotic lesions and ulcers, has been reported, largely anecdotely, in patients with the antiphospholipid syndrome and it has also been suggested that these manifestations might serve as early markers of the syndrome. ${ }^{10}$ The association of livedo reticularis with antiphospholipid antibodies has been confirmed in two controlled series of patients with SLE. ${ }^{11} 12$ In patients without SLE, the association, though plausible, has not been adequately documented by similar studies.

We have carried out a case-control study of the association of a variety of dermatological manifestations in which vascular abnormalities could play a major part-for example, livedo reticularis, acrocyanosis, and ulcers-with antiphospholipid antibodies. We limited our studies to patients without SLE and to patients at their first diagnosis of disorders possibly associated with antiphospholipid antibodies.

Patients and methods

The study was conducted in the period November 1988 to July 1990 on consecutive patients referred to the coagulation and 
haemostasis service of our general hospital for the first determination of antiphospholipid antibodies. Patients referred to the haematological centre had a variety of newly diagnosed disorders, including thrombocytopenia, thrombotic disorders, and unexplained repeated abortions (at least two consecutive miscarriages in the absence of clear aetiological factors). We were interested in studying the cutaneous disorders associated with early systemic manifestations suspected of being related to antiphospholipid antibodies in patients without SLE. For that reason, patients referred by dermatologists were excluded from the study, as were patients with a diagnosis of SLE at any time during the study period. Five patients were excluded because of a diagnosis of SLE.

For each patient lupus anticoagulant and anticardiolipin antibodies were investigated. All patients were subsequently examined by two dermatologists (LN, FL) who were unaware of the serological determinations; information was obtained about the patient's general characteristics (age, sex, occupation) and relevant dermatological and medical histories were collected by an ad hoc questionnaire. Strict criteria for physical examination were defined at the beginning of the study: all patients were examined at room temperature $\left(22-23^{\circ} \mathrm{C}\right.$ ) after at least an hour in the hospital environment.

Cutaneous manifestations looked for in the physical examination were livedo reticularis, acrocyanosis, erythrocyanosis, atrophie blanche, white porcelain scars, cutaneous ulcers, purpura, and capillaritis. In addition to these disorders, any other important cutaneous manifestations were recorded. Livedo reticularis was graded as mild, moderate, or severe based on the criteria developed by Weinstein $e t a l,{ }^{11}$ which take into account the extension and confluence of lesions and the intensity of bluish discoloration. Typical cases were presented and agreed upon at the start of the study. Histories of Raynaud's phenomenon, chilblains, cold urticaria, superficial thrombophlebitis, and cutaneous ulcers were collected. To be accepted, a diagnosis had to be made by a medical doctor and confirmed, when indicated (for example, cold urticaria), by appropriate tests. For doubtful cases, medical documentation was requested.

To determine lupus anticoagulant the following tests on platelet poor plasma were performed $^{13}:$ activated partial thromboplastin time with two different commercial reagents (activated Thrombofax (Ortho Diagnostic, Raritan, NI, USA) and activated partial thromboplastin time with kaolin activator (Boehringer Mannheim, Germany)), kaolin clotting time and diluted Russel viper venom time.

Platelet poor plasma was obtained by high speed centrifugation at $4^{\circ} \mathrm{C}$ and immediately stored at $-70^{\circ} \mathrm{C}$ until use. Platelet poor plasma from 20 normal subjects was pooled and used as a reference. When the ratio of the patient's clotting time to the control clotting time was higher than 1.3 in at least three tests and no correction was seen after 1:1 mixing of the patient's platelet poor plasma with normal platelet poor plasma, lupus anticoagulant was considered to be present.

IgG anticardiolipin antibodies were measured by an enzyme linked immunosorbent assay (ELISA). ${ }^{14}$ A standard curve was established with a reference serum. Results were reported in GPL standard units ${ }^{15}$ and normal values were lower than 10 units.

\section{ANALYSES OF DATA}

The study was analysed as a case-control study. Cases were patients with a positive test for lupus anticoagulant or anticardiolipin antibodies, or both. The controls were all other patients who were negative for lupus anticoagulant and anticardiolipin antibodies. Frequencies of cutaneous manifestations in cases and controls were compared; odds ratios (as estimators of relative risks) and their $95 \%$ confidence intervals were calculated by standard procedures ${ }^{16}$ and frequencies were compared by Fisher's exact test. All $p$ values were two tailed.

\section{Results}

Thirty five patients were examined. Thirteen patients had positive results for lupus anticoagulant or had increased titres of anticardiolipin antibodies, or both (cases). The other 22 patients had negative results (controls). In particular, 11 cases were positive for lupus anticoagulant and anticardiolipin antibodies; one case was positive for lupus anticoagulant only, and one case for anticardiolipin antibodies only. No patient was positive for only one or two of the tests used to look for lupus anticoagulant. The median titre of anticardiolipin antibodies was $74 \mathrm{U}$ $(8-190 \mathrm{U})$ for the cases and $7 \mathrm{U}(1-9 \mathrm{U})$ for the controls. Table 1 gives the general characteristics and referral diagnoses according to the serological status of the patients.

Table 2 gives the results of physical examinations. Moderate to severe livedo reticularis and acrocyanosis were significantly associated with antiphospholipid antibodies, with relative risks of $13 \cdot 1 \quad(95 \%$ confidence interval $1 \cdot 1$ to $149 \cdot 0)$ and $8 \cdot 6(95 \%$ confidence interval $1 \cdot 1$ to $65 \cdot 1$ ). Capillaritis was also significantly associated with antibodies (Fisher's exact test, $\mathrm{p}=0 \cdot 04$ ). To our surprise,

Table 1 General characteristics and referral diagnoses of the patients according to their serological status

\begin{tabular}{lll}
\hline Characteristic & \multicolumn{2}{l}{ Antiphospholipid antibodies } \\
\cline { 2 - 3 } & $\begin{array}{l}\text { Positive } \\
(n=13)\end{array}$ & $\begin{array}{l}\text { Negative } \\
(n=22)\end{array}$ \\
\hline Median age (range) (years) & $39(24-64)$ & $33(25-64)$ \\
Sex (M/F) & $3 / 10$ & $5 / 17$ \\
Diagnoses & & \\
Repeated abortions & 2 & 9 \\
Deep venous thromboses & 5 & 4 \\
Ischaemic stroke & 2 & 2 \\
Thrombocytopenia & 4 & 5 \\
Immunohaemolytic anaemia & 1 & 2 \\
Retroperitoneal haemorrhage & 1 & 0 \\
\hline
\end{tabular}

${ }^{\star}$ For two patients more than one diagnosis was made. 
Table 2 Distribution of cases and controls according to results of the physical examination

\begin{tabular}{llll}
\hline & $\begin{array}{l}\text { Cases* } \\
(n=13)\end{array}$ & $\begin{array}{l}\text { Controls } \\
(n=22)\end{array}$ & $\begin{array}{l}\text { Relative risk } \\
\text { (95\% confidence } \\
\text { interval) }\end{array}$ \\
\hline $\begin{array}{l}\text { Moderate to severe } \\
\text { livedo reticularis }\end{array}$ & 5 & & $13 \cdot 1(1 \cdot 1$ to $149 \cdot 0)$ \\
Acrocyanosis & 6 & 2 & $8 \cdot 6(1 \cdot 1$ to $65 \cdot 1)$ \\
Erythrocyanosis & 1 & 2 & $0 \cdot 8(0 \cdot 4$ to $1 \cdot 8)$ \\
Atrophie blanche & 1 & 1 & $1 \cdot 7(0 \cdot 0$ to $36 \cdot 6)$ \\
White porcelain scars & 1 & 0 & - \\
Capillaritis & 3 & 0 & $-t$ \\
\hline
\end{tabular}

${ }^{\star}$ Cases are positive for antiphospholipid antibodies and controls are negative for antiphospholipid antibodies.

†Fisher's exact test, $p=0 \cdot 04$.

Table 3 Distribution of cases and controls according to anamnestic inquiry. Only medically confirmed diagnoses were accepted

\begin{tabular}{llll}
\hline & $\begin{array}{l}\text { Cases } \\
(n=13)\end{array}$ & $\begin{array}{l}\text { Controls } \\
(n=22)\end{array}$ & $\begin{array}{l}\text { Fisher's exact } \\
\text { test: } p \text { value }\end{array}$ \\
\hline Raynaud's phenomenon & 3 & 0 & 0.04 \\
Chilblains & 6 & 8 & NS \\
Superficial thrombophlebitis & 3 & 0 & 0.04 \\
Cutaneous ulcers & 2 & 0 & NS \\
\hline
\end{tabular}

*Cases are positive for antiphospholipid antibodies and controls are negative for antiphospholipid antibodies. example, selection of cases (or controls) according to some characteristic they show which is related to the risk factor under evaluation ${ }^{16}$-we excluded patients referred by a dermatologist from the study. We also excluded patients with SLE, and even assuming the possibility that the referring doctor might have predicted, to some extent, the serological status of patients, it seems unlikely that the patient was selectively referred to our hospital for the determination of antiphospholipid antibodies because of the presence of a relatively subtle cutaneous manifestation such as that studied here. A final methodological issue of our study regards its 'power'. For disorders with a low prevalence $(10 \%$ or less) in the control group, our study was designed to detect increases in risk of tenfold or more over the controls, while less dramatic increases in risk could not be detected.

Although the cutaneous disorders examined appear to be a heterogeneous group, they have the common characteristic of all being considered to be related to primary vascular abnormalities, functional or thrombotic. Livedo reticularis is a violet discoloration of the skin with a reticular or cobblestone appearance. It is considered to result from disordered blood flow through subpapillary and dermal blood vessels. ${ }^{17}$ 18 Mild livedo reticularis - that is, light purple discoloration mostly limited to the legs-is considered to be a normal variant, common in young women, whereas moderate to severe livedo reticularisthat is, livedo with a darker purple discoloration affecting the arms and legs, and sometimes other areas-has been described in many diseases including connective tissue diseases. The association of moderate to severe livedo reticularis and antiphospholipid antibodies has been documented in two previous controlled series of patients with SLE. ${ }^{11} 12$ We have confirmed the results of those studies for patients without SLE. The prevalence of moderate to severe livedo reticularis in patients with antiphospholipid antibodies was $33 \%$ in the study of Weinstein et $a l,{ }^{11}$ and was $25 \%$ in our study.

Acrocyanosis refers to dusky discoloration of the hands and feet, with an abnormal sensitivity to cold. ${ }^{12}$ The underlying mechanism is unknown but a disordered flow in the veins and changes in blood viscosity have been suggested. ${ }^{19}$ To the best of our knowledge acrocyanosis has not been reported previously in association with antiphospholipid antibodies. The association of this disorder with livedo reticularis is well known, however.

Capillaritis is a general term that indicates several chronic disorders, including Schamberg's disease, eczematide-like purpura, Majocchi's disease, and pigmented purpuric lichenoid dermatosis of Gougerot and Blum, characterised by purpuric lesions and pigmentary changes with time. Often, as in our patients, only a generic clinical diagnosis of capillaritis is possible. ${ }^{20}$ Common histological features include extravasation of red cells, deposits of haemosiderin, and alteration of status of the patients. Our study could regarded as a case-control study with skin manifestations being the risk factor analysed and the antiphospholipid antibody status determining whether a patient was a case (positive results) or a control (negative results). To avoid a potential selection bias-for 
small superficial vessels (endothelial swelling, perivascular lymphocytic infiltrate). Gravity and increased venous pressure are considered important localising factors. Although purpura has been reported as a cutaneous manifestation in patients with antiphospholipid syndrome, capillaritis, to our knowledge, has not been reported previously.

Superficial thrombophlebitis is a well known manifestation of the antiphospholipid syndrome, as is deep vein thrombosis, and it was not surprising that a history of this disorder was considerably more common in our patients positive for antiphospholipid antibodies. Raynaud's phenomenon is characterised by pallor followed by cyanosis of the hands and sometimes feet precipitated by cold and due to paroxysmal constriction of arterioles. ${ }^{17}{ }^{21} \mathrm{It}$ has been described in several disorders including connective tissue, haematological (for example, cold haemoagglutinins), and neurovascular disorders (for example, shoulder girdle compression syndrome). The aetiology of idiopathic cases is still unknown but primary alterations in blood flow, including increased blood viscosity, platelet aggregation, and impaired fibrinolysis, have been considered. ${ }^{21}$ Systemic sclerosis, a disorder in which Raynaud's phenomenon is prominent, has been described in association with antiphospholipid antibodies..$^{22}$

Neurofibromatosis type I, malignant melanoma, and widespread angiomata in patients in our study with antiphospholipid antibodies remain unexplained, and even if these might be a chance occurrence, in our opinion they merit further study in view of the rarity of the disorders. ${ }^{24}$

In conclusion, our study provides quantitative evidence of the association of antiphospholipid antibodies with several cutaneous manifestations, suggesting that these manifestations might appear early in the development of the antiphospholipid syndrome. The lesions we observed were subtle; more dramatic events, such as cutaneous ulcers, often reported as clinical manifestations of the antiphospholipid syndrome, are probably either rarer than previously considered or appear later in the development of the syndrome.
The reference serum for anticardiolipin antibodies was kindly provided by $\operatorname{Dr} \mathrm{E} N$ Harris (London)

1 Harris E N, Gharavi A E, Hughes G R V. Antiphospholipid antibodies. Clin Rheum Dis 1985; 11: 591-609.

2 Hughes, G R V, Harris E N, Gharavi A E. The anticardiolipin syndrome. F Rheumatol 1986; 13: 486-9.

3 Asherson R A, Cervera R. The antiphospholipid syndrome: a syndrome in evolution. Ann Rheum Dis 1992; 51: 147-50.

4 Hamsten A, Norberg R. Antibodies to phospholipids in thrombotic disease. F Intern Med 1989; 225: 363-5.

5 Hamsten A, Norberg R, Biorkholm M, de Faire U, Holm G. Antibodies to cardiolipin in young survivors of myocardial infarction: an association with recurrent cardiovascular events. Lancet 1986; i: 113-6.

6 Morton K E, Gavaghan T P, Krilis S A et al. Coronary artery bypass graft failure - an autoimmune phenomenon? Lancet 1986; ii: 1353-7.

7 Sturfelt G, Nived O, Norberg R, Thorstensson R, Krook K. Anticardiolipin antibodies in patients with systemic lupus erythematosus. Arthritis Rheum 1987; 30: 382-8.

8 Sontheimer R D. The anticardiolipin syndrome: a new way to slice on old pie, or a new pie to slice? Arch Dermatol to slice on old pie,

9 Stephens J M C. The antiphospholipid syndrome. Clinical correlations, cutaneous features, mechanism of thrombosis and treatment of patients with the lupus anticoagulant and anticardiolipin antibodies. $\mathrm{Br} f$ Dermatol 1991; 125: 199-210

10 Alegre V A, Gastineau D A, Winkelmann R K. Skin lesions associated with circulating lupus anticoagulant. $\mathrm{Br} \mathcal{F}$ Dermatol 1989; 120: 419-29.

11 Weinstein C, Miller M H, Axtens R, et al. Livedo reticularis associated with increased titres of anticardiolipin antibodies in systemic lupus erythematosus. Arch antibodies in systemic lup

12 Englert H J, Loizou S, Derue G G M, et al. Clinical and immunologic features of livedo reticularis in lupus: a casecontrol study. Am F Med 1989; 87: 408-10.

13 Galli M, Comfurius P, Maassen C, et al. Anticardiolipin antibodies (ACA) directed not to cardiolipin but to a plasma protein cofactor. Lancet 1990; 335: 1544-7.

14 Loizou S, McCrea J D, Rudge A C, et al. Measurement of anticardiolipin antibodies by an enzyme-linked immunoadsorbent assay (ELISA): standardization and quantitation of results. Clin Exp Immunol 1985; 62: 738-45.

15 Harris E N, Hughes G R V. Standardising the anticardiolipin antibody test. Lancet 1987; i: 277.

16 Breslow N E, Day, N E. Statistical methods in cancer research. Vol. I. The analysis of case-control studies. Lyon: IARC Scientific Publications, 1980.

17 Page H E, Shear N H. Temperature-dependent skin disorders. F Am Acad Dermatol 1988; 18: 1003-19.

18 Copeman P W M. Livedo reticularis. Br f Dermatol 1975 93: 519-29.

19 Ryan T J, Copeman P W M. Microvascular pattern and blood stasis in skin disease. $B r f$ Dermatol 1969; 81: 563-73.

20 Champion R H. Purpura. In: Rook A, Wilkinson D S Ebling F J G, Champion R H, Burton J L, eds. Textbook of dermatology. 4th ed. Oxford: Blackwell Scientific, 1986: 1116-9.

21 Dowd P M. Cold-related disorders. Prog Dermatol 1986; 20: 1-8.

22 Malia R G, Greaves M, Rowlands A C, et al. Anticardiolipin antibodies in systemic sclerosis: immunological and clinical associations. Clin Exp Immunol 1988; 73: 456-60.

23 Katayama I, Otoyama K, Kondo S. Clinical manifestations in anticardiolipin antibody-positive patients with progressive systemic sclerosis. $₹$ Am Acad Dermatol 1990; 23: 198-201.

24 Naldi L, Finazzi G, Brevi A, et al. Antiphospholipid antibodies and melanoma: a link? Dermatology 1992; 184; 156. 